Supporting Information

\title{
Impact of Plasmonic Photothermal Effects on the Reactivity of Au Nanoparticle Modified Graphene Electrodes Visualized using Scanning Electrochemical Microscopy
}

Noah B. Schorr, Michael J. Counihan, Rohit Bhargava, Joaquín Rodríguez-López*

\section{Table of Contents:}

Figure S1. Geometry and associated equations for finite-elements simulations.

Page S2

Figure S2. Simulated results for substrate-tip and heating elements size.

Page S3

Figure S3. Results of ORR performed at the SECM tip over G-AuNP.

Page S3

Figure S4. CV of G-AuNP taken with various laser powers.

Page S4

Figure S5. ORR at the SECM tip at various laser powers.

Page S4

Figure S6. Images of 10-layer G-AuNP

Page S5

Figure S7. SECM imaging of 10-layer G-AuNP.

Page S5

Figure S8. Tip collection curves for ORR at single and 10-layer graphene. 


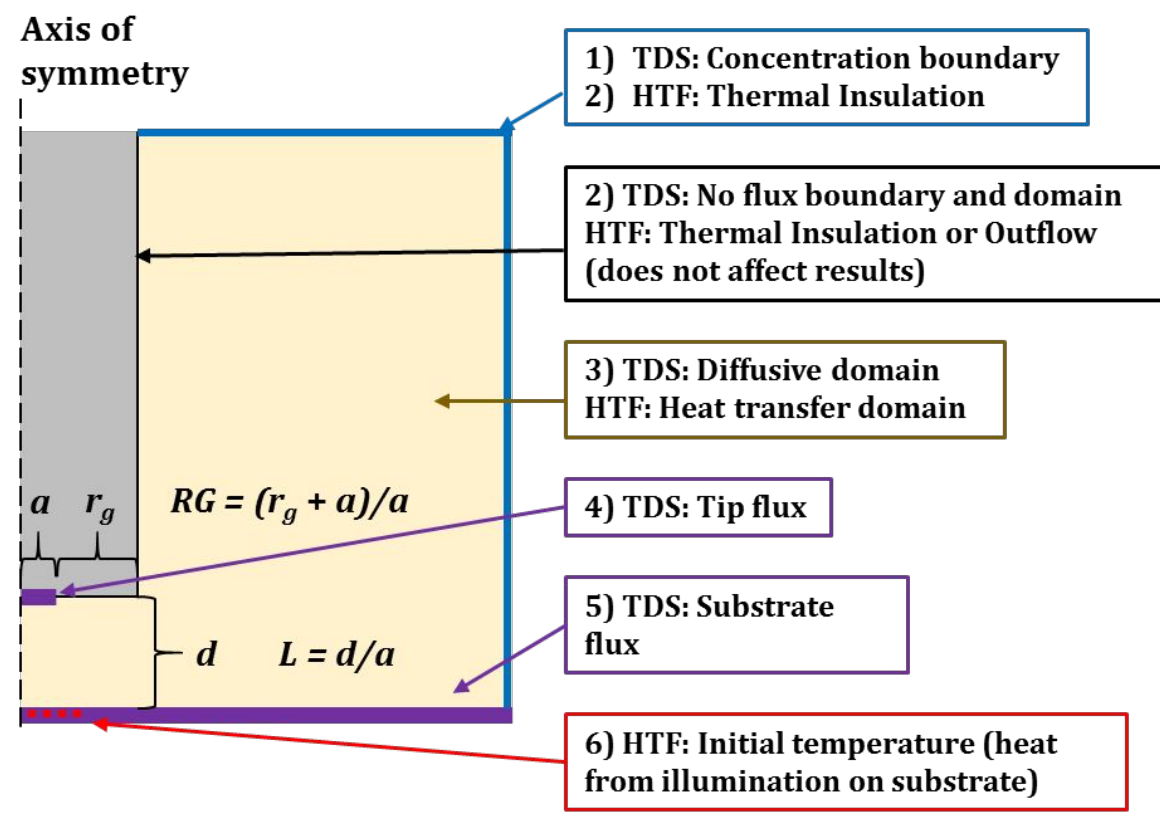

Relevant expressions

1) $[0]=[0]_{0},[R]=[R]_{0},[\mathrm{HP}]=\mathbf{0}$

\begin{tabular}{|c|c|c|}
\hline 3) & $\begin{array}{c}-J_{i}(x, t)=D_{i} \frac{\partial C_{i}(x, t)}{\partial x} \\
\frac{\partial C_{i}(x, t)}{\partial x}=D_{i}\left(\frac{\partial^{2} C_{i}(x, t)}{\partial x^{2}}\right) \\
D_{i}=\frac{k_{\text {boltz }} \times T}{6 \pi r_{i} \times \eta_{\text {sol }}} \\
r_{\mathrm{FcMeOH}}=350 \mathrm{pm} \\
r_{\mathrm{O}_{2}}=122 \mathrm{pm} \\
r_{\mathrm{HP}}=200 \mathrm{pm}\end{array}$ & $\begin{array}{l}\text { All temperature-dependent properties of } \\
\text { water, calculated empirically by COMSOL: } \\
k \text { - Thermal conductivity } \\
\rho \text { - Density } \\
C_{p} \text { - Heat capacity at } 1 \text { atm pressure } \\
\gamma \text { - Ratio of specific heats } \\
T_{\text {initial }}-298 \mathrm{~K} \\
\eta_{\text {sol }}=1.3799566804-(0.021224019151 \times \\
\left.T^{-1}\right)+\left(\left(1.3604562827 \times 10^{-4}\right) \times T^{-2}\right) \ldots\end{array}$ \\
\hline
\end{tabular}

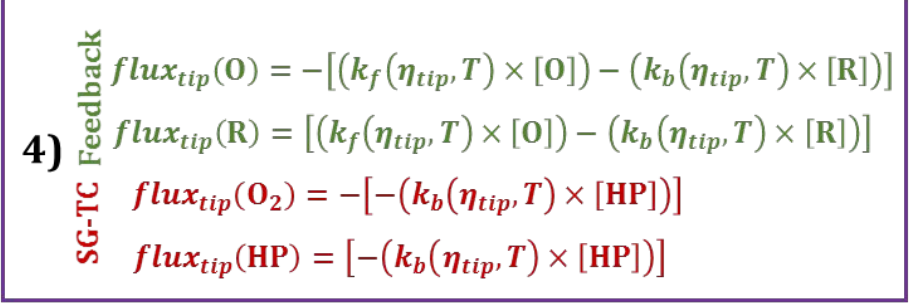
$i_{\text {tip }}=\operatorname{flux}_{\text {tip }}(0) \times n F \times \pi a^{2}$
$4 \& 5) k_{f}(\eta, T)=k^{0} \times \exp \left(\frac{-\alpha F \eta}{R T}\right)$
$k_{b}(\eta, T)=k^{0} \times \exp \left(\frac{(1-\alpha) F \eta}{R T}\right)$

6) $T_{0}$ - set temperature

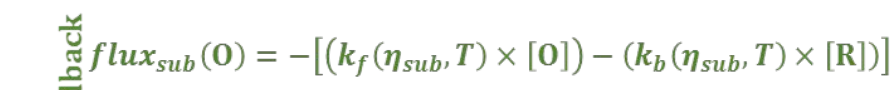

5) d $_{\text {i. }} \operatorname{lux}_{s u b}(\mathrm{R})=\left[\left(k_{f}\left(\eta_{s u b}, T\right) \times[0]\right)-\left(k_{b}\left(\eta_{s u b}, T\right) \times[R]\right)\right]$

战 $\operatorname{flux}_{\text {sub }}\left(\mathrm{O}_{2}\right)=-\left[\left(\boldsymbol{k}_{f, H P}\left(\eta_{\text {sub }}, T\right) \times\left[\mathrm{O}_{2}\right]\right)\right]$

i $\operatorname{flux}_{\text {sub }}(\mathrm{HP})=\left[\left(k_{f, H P}\left(\eta_{s u b}, T\right) \times\left[O_{2}\right]\right)\right]$
}

Figure S1. Geometry and associated equations for finite-elements simulations. Transport dilute species module $=$ TDS and heat transfer in fluids module $=$ HTF. 

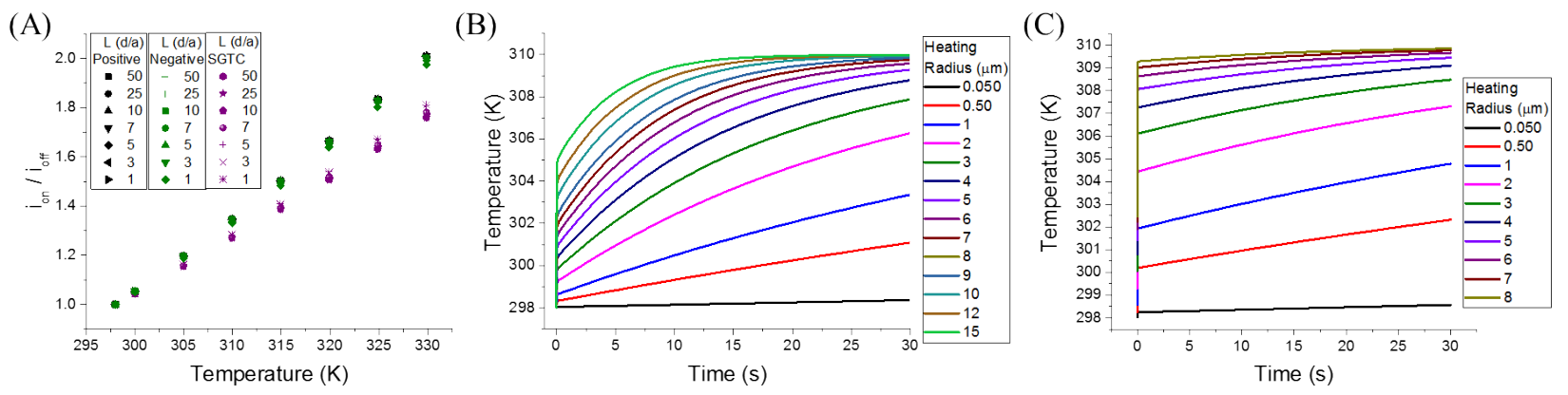

Figure S2. Simulated results for substrate-tip and heating elements size. (A) Plot of $i_{\text {on }} / i_{\text {off }}$ versus temperature for a positive feedback, negative feedback, and SGTC at varying L, which is the normalized parameter for distance between tip and substrate normalized by the tip radius. (B) Temperature at tip over time when the size of the heating element is varied and tip is at $5 \mathrm{~L},(\mathrm{C})$ $1 \mathrm{~L}$.

(A)
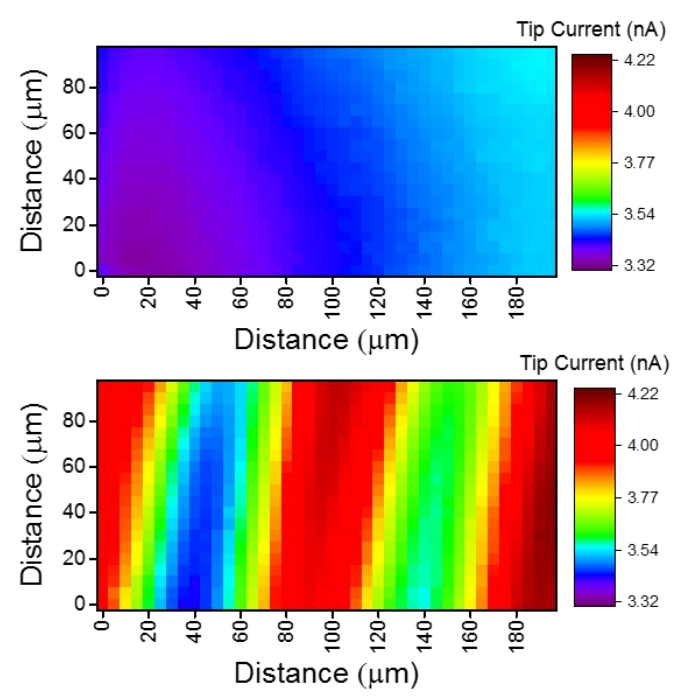

(B)

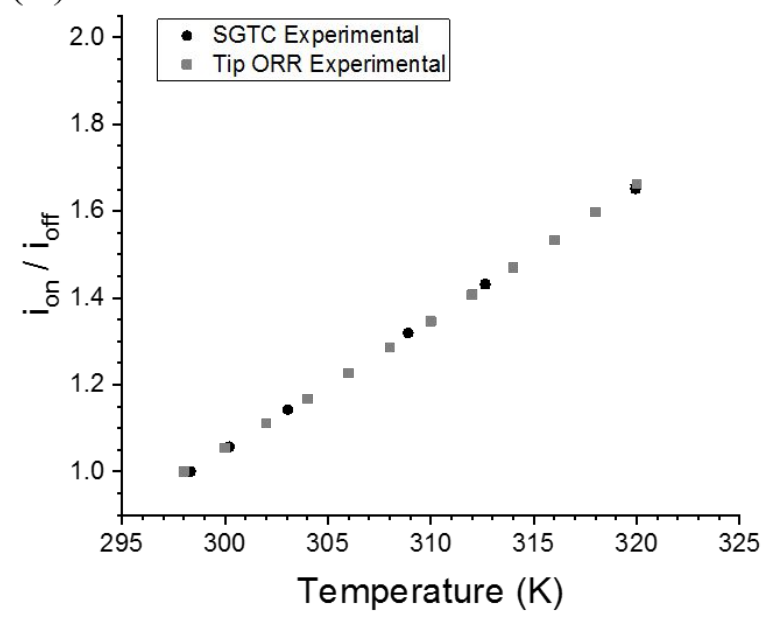

Figure S3. Results of ORR performed at the SECM tip while the G-AuNP remained unbiased. (A) Top, SECM using ORR at the UME over the G-AuNP showing only negative feedback. Bottom, SECM with light excitation. (B) Calibration between experimental $i_{\text {on }} / i_{\text {off }}$ values at varying laser power and finite-element simulated temperatures SECM tip held at $-0.55 \mathrm{~V}$ vs $\mathrm{Ag} / \mathrm{AgCl}$ and substrate biased at OCP for tip ORR experiments 


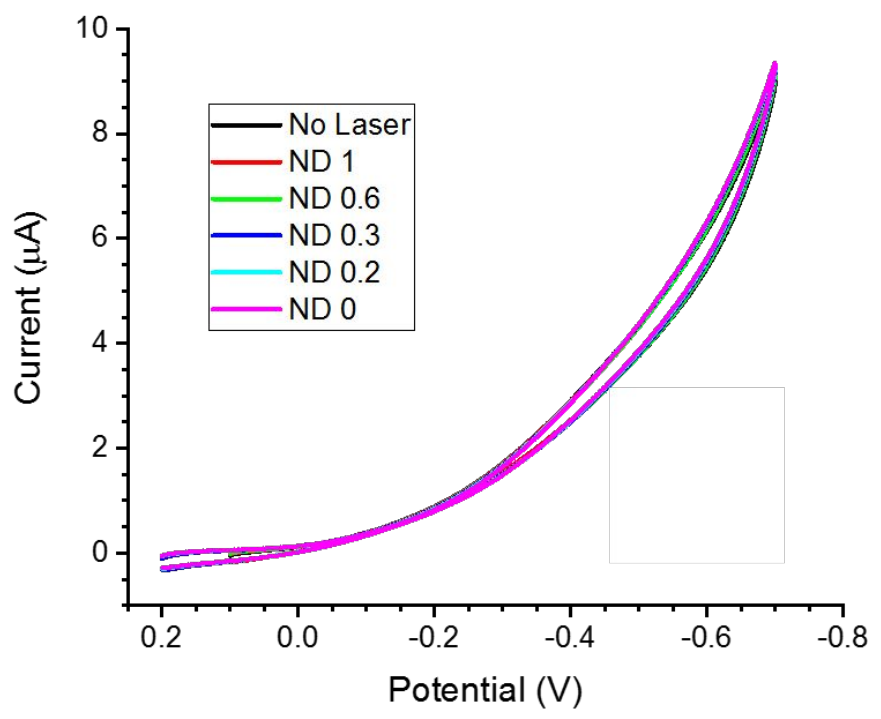

Figure S4. Cyclic voltammogram of G-AuNP in $100 \mathrm{Mm} \mathrm{Na}_{2} \mathrm{SO}_{4}$ taken with various laser powers.

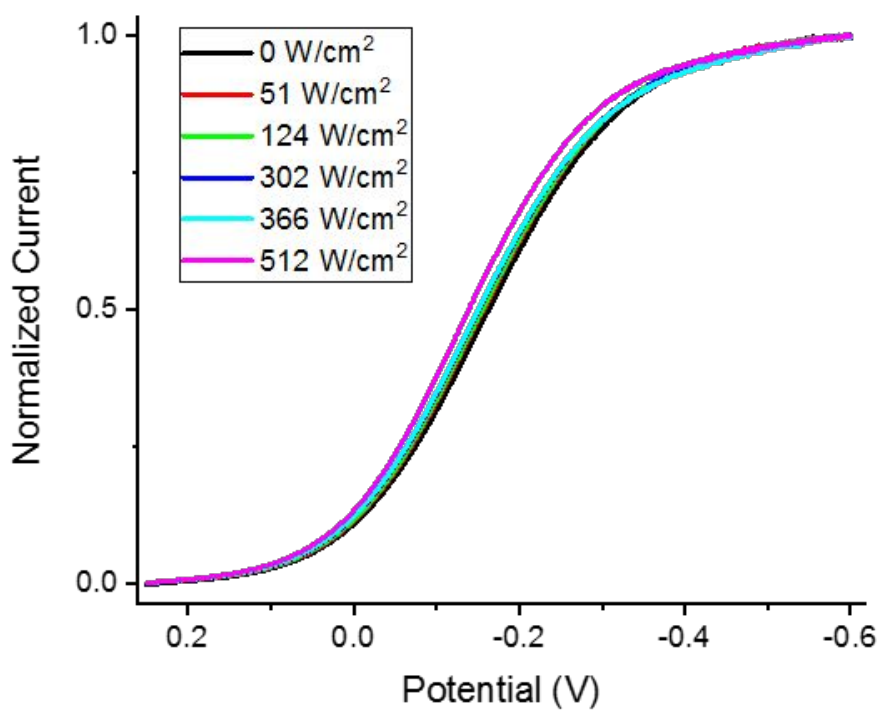

Figure S5. ORR at the SECM tip at various laser powers with a maximum shift of $24 \mathrm{mV}$. 
(A)

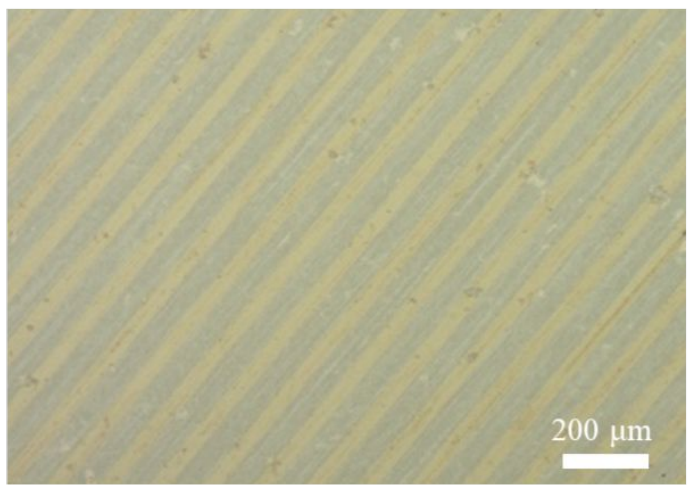

(B)

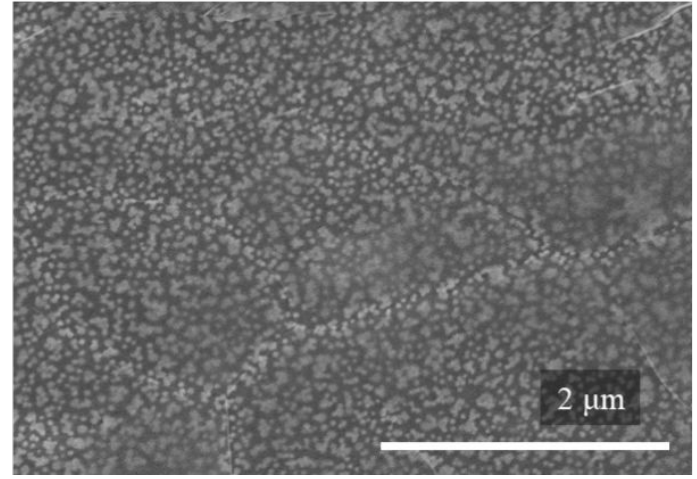

Figure S6. 10L-G-AuNP sample. (A) Optical image of 10L-G-AuNP. (B) SEM images of 10LG-AuNP.
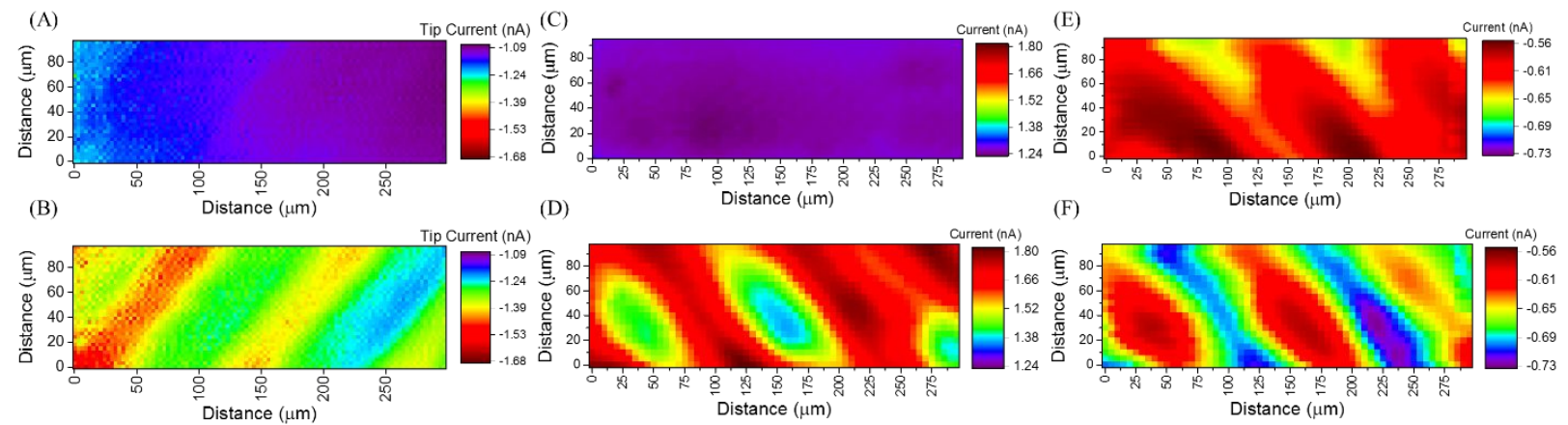

Figure S7. SECM imaging of 10-layer G-AuNP. (A) Tip current for FcMeOH feedback without laser. (B) Tip current for $\mathrm{FcMeOH}$ feedback with laser. Tip held at $0.425 \mathrm{~V}$ and substrate -0.15 $\mathrm{V}$ vs $\mathrm{Ag} / \mathrm{AgCl}$ for feedback imaging. (C) Tip ORR without laser. (D) With laser illumination. Tip held at $0.425 \mathrm{~V}$ vs $\mathrm{Ag} / \mathrm{AgCl}$ and substrate at OCP for tip ORR imaging. (E) SGTC without laser. (F) SGTC with laser. Tip held at $1.00 \mathrm{~V}$ and substrate $-0.65 \mathrm{~V} \mathrm{vs} \mathrm{Ag} / \mathrm{AgCl}$ for SGTC imaging. 

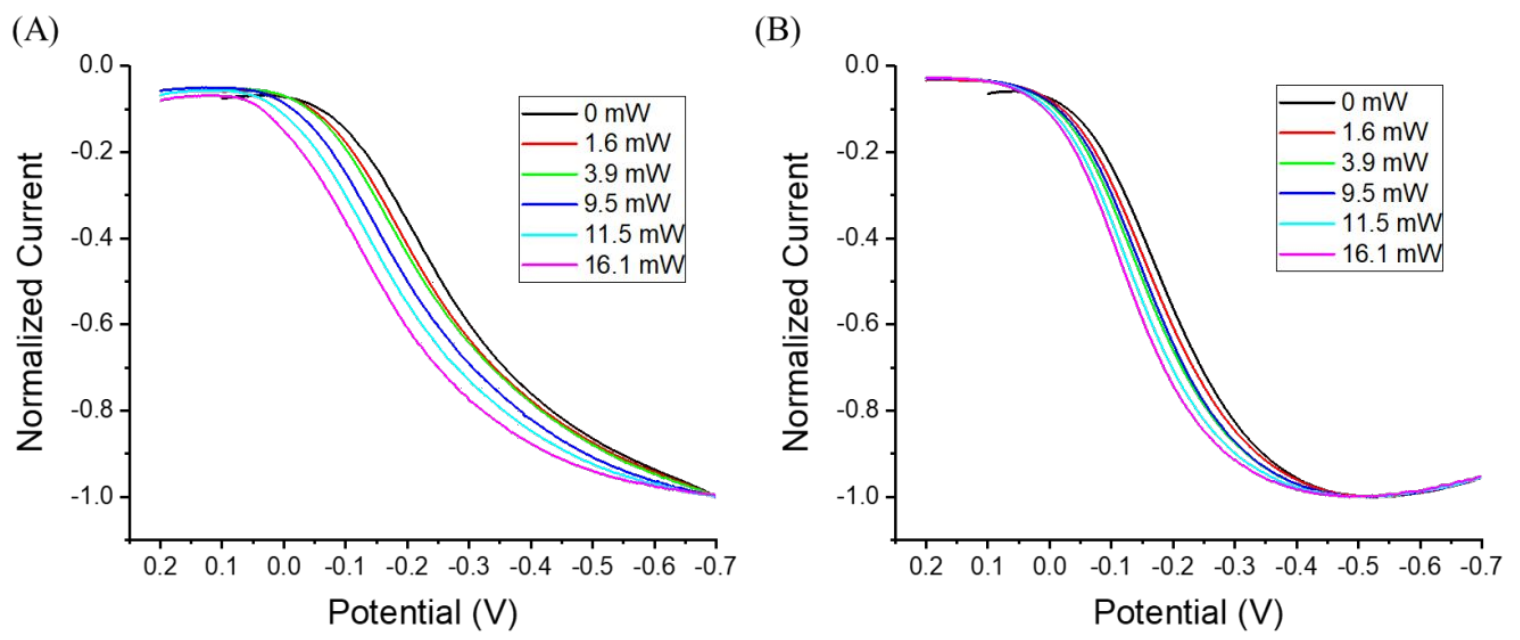

Figure S8. Tip collection curves during substrate generation. (A) Normalized current at the tip associated with the ORR at the G-AuNP substrate with increasing laser power. (B) Normalized current at the tip associated with the ORR at the 10-G-AuNP substrate with increasing laser power. 